\section{(C) OPEN ACCESS}

\title{
Scleroderma fibroblasts suppress angiogenesis via TGF- $\beta$ /caveolin-1 dependent secretion of pigment epithelium-derived factor
}

\author{
Vasiliki Liakouli, ${ }^{1,2}$ Jacobo Elies, 1,3 Yasser Mohamed El-Sherbiny, 1,4,5 \\ Margherita Scarcia, ${ }^{6}$ Gary Grant, ${ }^{6}$ Giuseppina Abignano, ${ }^{1,7,8}$ Emma C Derrett-Smith, ${ }^{9}$ \\ Filomena Esteves, ${ }^{10}$ Paola Cipriani, ${ }^{2}$ Paul Emery, ${ }^{1,5}$ Christopher P Denton, ${ }^{9}$ \\ Roberto Giacomelli, ${ }^{2}$ Georgia Mavria, ${ }^{6}$ Francesco Del Galdo ${ }^{1,5}$
}

\begin{abstract}
Handling editor Tore K Kvien
- Additional material is published online only. To view please visit the journal online (http://dx.doi.org/10.1136/ annrheumdis-2017-212120).
\end{abstract}

For numbered affiliations see end of article.

\section{Correspondence to}

Dr Georgia Mavria, Signal Transduction and Tumour Microenvironment Group, Leeds Institute of Cancer and Pathology, University of Leeds, Leeds, LS2 9JT, UK; g.mavria@leeds.ac.uk and Dr Francesco Del Galdo, NIHR Leeds Musculoskeletal Biomedical Research Centre, Leeds Teaching Hospital NHS Trust, Leeds LS74SA, UK; f.delgaldo@leeds.ac.uk

VL and JE contributed equally.

Received 24 July 2017 Revised 21 November 2017 Accepted 29 November 2017 Published Online First 19 December 2017

\section{ABSTRACT}

Objectives Systemic sclerosis (SSC) is characterised by tissue fibrosis and vasculopathy with defective angiogenesis. Transforming growth factor beta (TGF- $\beta$ ) plays a major role in tissue fibrosis, including downregulation of caveolin-1 (Cav-1); however, its role in defective angiogenesis is less clear. Pigment epithelium-derived factor (PEDF), a major antiangiogenic factor, is abundantly secreted by SSc fibroblasts. Here, we investigated the effect of TGF- $\beta$ and Cav- 1 on PEDF expression and the role of PEDF in the ability of SSC fibroblasts to modulate angiogenesis.

Methods PEDF and Cav-1 expression in fibroblasts and endothelial cells were evaluated by means of immunohistochemistry on human and mouse skin biopsies. PEDF and Cav-1 were silenced in cultured SSC and control fibroblasts using lentiviral short-hairpin RNAs. Organotypic fibroblast-endothelial cell cocultures and matrigel assays were employed to assess angiogenesis.

Results PEDF is highly expressed in myofibroblasts and reticular fibroblasts with low Cav-1 expression in SSc skin biopsies, and it is induced by TGF- $\beta$ in vitro. SSc fibroblasts suppress angiogenesis in an organotypic model. This model is reproduced by silencing Cav-1 in normal dermal fibroblasts. Conversely, silencing PEDF in SSc fibroblasts rescues their antiangiogenic phenotype. Consistently, transgenic mice with TGF- $\beta$ receptor hyperactivation show lower Cav-1 and higher PEDF expression levels in skin biopsies accompanied by reduced blood vessel density.

Conclusions Our data reveal a new pathway by which TGF- $\beta$ suppresses angiogenesis in SSc, through decreased fibroblast Cav-1 expression and subsequent PEDF secretion. This pathway may present a promising target for new therapeutic interventions in SSC.

\section{INTRODUCTION}

Systemic sclerosis (SSc) or scleroderma is an autoimmune connective tissue disease characterised by accumulation of extracellular matrix (ECM) proteins within the affected tissues and a widespread vasculopathy comprising both defective angiogenesis and fibroproliferative vasculopathy. ${ }^{1-4}$ It is well established that transforming growth factor beta (TGF- $\beta$ ) plays a key role in accumulation of collagen and ECM proteins and downregulation of caveolin-1 (reviewed in Del Galdo et al $^{5}$ and Lafyatis ${ }^{6}$ ). Nevertheless, the pathogenesis of sustained microangiopathy and defective angiogenesis, and their causal links with tissue fibrosis are less clearly understood. Recent studies employing a mouse strain with a ligand-dependent upregulation of TGF- $\beta$ signalling in tissue fibroblasts (TBRII $\Delta \mathrm{k}$-fib) demonstrate that increased TGF- $\beta$ signalling favours the onset of fibroproliferative vasculopathy typical of SSc following minimal endothelial cell injury, suggesting that TGF- $\beta$ activation may also play a role in the pathogenesis of vasculopathy in SSc.

Proteomic studies have previously identified pigment epithelial-derived factor (PEDF) as one of the most abundant secreted proteins by SSc skin fibroblasts compared with healthy controls. ${ }^{8}$ PEDF is a $46 \mathrm{kDa}$ secreted glycoprotein that belongs to the serpin superfamily but has no protease inhibitory function. Despite the lack of protease activity, PEDF exerts diverse physiological functions including antiangiogenesis, ${ }^{9}$ antivasopermeability ${ }^{10}$ and neurotrophic activities. ${ }^{11} 12$ PEDF is expressed abundantly in pigmented epithelium of the cornea where it plays a crucial role in the suppression of angiogenesis. ${ }^{9} 13-16$

Previous studies have shown that PEDF is highly expressed in idiopathic pulmonary fibrosis and is inducible by TGF- $\beta$ in cultured human lung fibroblasts, suggesting a potential link with the pathogenesis of tissue fibrosis. ${ }^{17}$ Here, we set to determine PEDF expression in SSc skin biopsies and in TBRII $\Delta \mathrm{k}$-fib mice in vivo and to determine the role of PEDF expression in the antiangiogenic function of fibroblasts in vitro.

\section{MATERIALS AND METHODS}

\section{Patient samples and patient skin biopsies}

Skin biopsies from nine patients with early diffuse cutaneous systemic sclerosis $(\mathrm{dcSSc})^{18}$ and nine healthy controls were obtained at the SSc clinic within the Leeds Institute of Rheumatic and Musculoskeletal Medicine (UK) and the Rheumatology Unit in L'Aquila (Italy). Biopsies were taken with full informed consent as approved by National Research Ethics Service (NRES) Committee (REC $10 / \mathrm{H} 1306 / 88$ ) and the local ethical committee in University of L'Aquila, and processed as described in detail in online supplementary methods. 


\section{Cell culture}

Dermal fibroblasts (FBs) from patients with dcSSc and controls were isolated as previously described ${ }^{19}$ and detailed in online supplementary methods. Human dermal microvascular endothelial cells (MVECs) were purchased from PromoCell, UK, cultured in Endothelial Cell Growth Medium BulletKit (Lonza, Slough, UK) and used at passages 2-4. Human umbilical vein endothelial cells (HUVECs) were obtained from TCS Cellworks, cultured in fully supplemented human large vessel endothelial cell medium (TCS Cellworks, UK) and used at passages 3-4. Human epidermal melanocytes (HEMs) were purchased from ScienceCell, cultured in melanocyte medium supplemented with melanocyte growth supplement (TCS Cellworks) and used at passages $3-4$. All cells were kept at $37^{\circ} \mathrm{C}$ in a humidified atmosphere of $5 \% \mathrm{CO}_{2}$.

\section{hTERT immortalisation and transduction with lentiviral short- hairpin RNA}

For immortalisation of primary FBs, pBabe human telomerase reverse transcriptase (hTERT) puromycin retrovirus was employed following standard protocols (described in detail in online supplementary methods). Cav-1 and PEDF expression were silenced by transduction with shRNAmir GIPZ lentiviruses (Open Biosystems, Surrey, UK) following manufacturer's instructions (online supplementary methods).

\section{Culture treatments}

To evaluate the effect of TGF- $\beta$ on PEDF and COLA1, cells were grown to confluence in six-well culture plates in dulbecco modified eagle medium (DMEM) 10\% fetal calf serum (FCS), serum starved in DMEM $0.5 \%$ FCS for 24 hours and stimulated in the presence of $10 \mathrm{ng} / \mathrm{mL}$ recombinant human (rh) TGF- $\beta 1$ (Sigma, USA) for 48 hours. Ascorbic acid $(40 \mu \mathrm{g} / \mathrm{mL})$ was used to optimise collagen production. ${ }^{20}$

\section{RNA isolation and RT-PCR analysis}

Total RNA was isolated using the RNeasy Mini Kit (Qiagen, USA) according to the manufacturer's instructions. One microgram of total RNA from each sample was retrotranscribed to first-strand cDNA using the SuperScript III One Step RT-PCR system (Invitrogen, UK). Quantitative RT-PCR was performed in triplicates using SYBR Green RT-PCR Master Mix Kit and the ABI PRISM 7500 Fast Real Time PCR System (Applied Biosystems) with the following primers: PEDF 5'-TGTCTCCAACTTCGGCTATG-3' (forward) and 5'-AGTAGAGAGCCCGGTGAATG-3' (reverse), Cav-1 5'-CGACCCTAAACACCTCAACGA-3' (forward) and 5'-TCCCTTCTGGTTCTGTCA-3' (reverse). Quantification was performed using the comparative $\mathrm{C}_{\mathrm{T}}$ (cycle-threshold) method employing ribosomal $18 \mathrm{~S}$ as a housekeeping gene.

\section{Protein quantification and immunodepletion}

Secreted PEDF and Collagen I were detected by immunoblotting in cultured cells and supernatants and by ELISA in patient sera as described in online supplementary methods. Immunodepletion for PEDF was conducted using monoclonal mouse anti-PEDF (Chemicon-Millipore, Clone 10F12.2). Full experimental procedures are described in detail in online supplementary methods.

\section{Angiogenesis assays}

The organotypic co-culture assay ${ }^{21-23}$ was performed with MVECs or HUVEC and either primary or hTERT immortalised fibroblasts stably infected with lentivirus coding for either non-silencing control, Cav-1 or PEDF short-hairpin (sh) RNAs as described in detail in online supplementary methods. Number of tubules and total tubule length were analysed using the Angiosys software (TCS Cellworks).

Reduced growth factor matrigel (VWR, UK) was used to perform angiogenesis matrigel assays in HUVEC employing supernatants from cultured FBs (online supplementary methods).

\section{FACS analysis and proliferation assay}

For PEDF detection using FACS analysis, fibroblasts were incubated in the presence of a protein transport inhibitor GolgiPlug (BD Biosciences) for 12 hours according to manufacturer's instructions. The cells were then stained on ice with rabbit polyclonal anti-PEDF-PECy5.5 (Bioss) following fixation and permeabilisation, and analysed against the corresponding isotype control using BD FACSDiva software V.6.0 BDTM LSR II flow cytometer.

For determination of HUVEC proliferation, cells were labelled with the carbocyfluorescein succinimidyl ester dye analogue, CellTrace Violet (Invitrogen). Prior to co-culture experiments, in order to track cell division following co-culture and for accurate gating, CD90-PEvio770 and CD31-APC (Miltenyi biotec) were used to exclude potential contamination of HUVEC with co-cultured fibroblasts; 7-aminoactinomycin D was used as a viability marker. Cell division frequency and proliferation indices from list mode data were determined using proliferation wizard of ModFit software V.3.2 (Verity Software House, Topsham, ME, USA).

\section{Immunohistochemistry}

Immunohistochemistry (IHC) analysis of human skin biopsies was performed on $3 \mu \mathrm{m}$ paraffin sections using mouse monoclonal anti-PEDF (Clone 10F12.2; Millipore, UK), rabbit polyclonal anti-CD31, rabbit polyclonal anti- $\alpha$-smooth muscle actin (SMA) (Abcam, UK) and a rabbit polyclonal anti-Cav-1 antibody (Santa Cruz, UK). Detailed procedure of the two-step staining is described in the online supplementary methods. The number of positive cells was counted by two pathologists, blinded to tissue source and expressed as the mean of two observations for each sample. For mouse skin biopsies, we employed rabbit polyclonal anti-PEDF (Aviva Systems Biology; Insight Biotech, UK), rabbit polyclonal anti-CD31 (Santa Cruz) and rabbit polyclonal anti-Cav-1 (Santa Cruz) antibodies. All sections were imaged using an Axioplan Zeiss light microscope equipped with an AxioCam digital camera.

\section{$T \beta R I I \Delta k$-fib animal model}

$\mathrm{T} \beta \mathrm{RII} \Delta \mathrm{k}$-fib transgenic mice were provided by Professor C. Denton of UCL Medical School Centre for Rheumatology and Connective Tissue Diseases, London, UK. The generation of $\mathrm{T} \beta \mathrm{RII} \Delta \mathrm{k}$-fib transgenic (TG) mice has been described previously. $^{7}$ Mice were genotyped by PCR using LacZ primers and an internal control. Experiments were performed on three transgenic mice aged 6 weeks and compared with sex-matched littermate controls. Animals were housed in a conventional clean facility, with access to food and water ad libitum. Strict adherence to institutional guidelines was practised, under full local ethics committee and Home Office approval. 
A
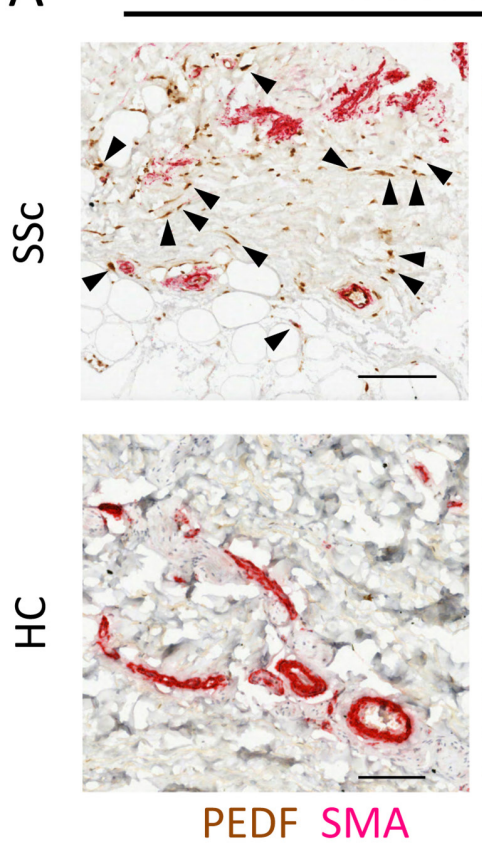

B

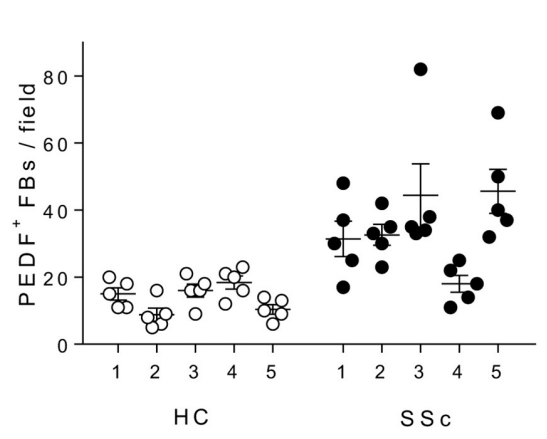

D

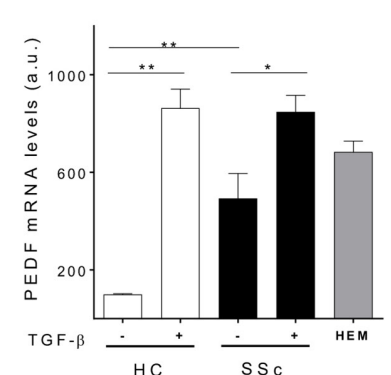

\section{Upper dermis}
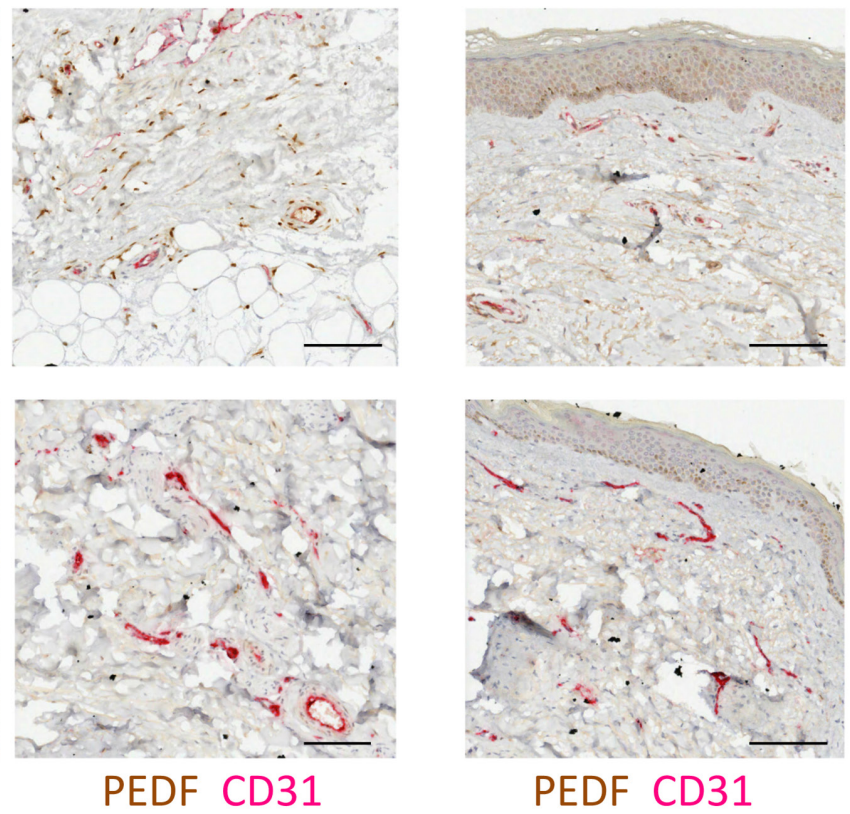

Figure 1 Pigment epithelium-derived factor (PEDF) expression is increased in systemic sclerosis (SSc) skin in vivo and it is inducible by transforming growth factor beta (TGF- $\beta$ ) in vitro. (A) Images depict representative forearm skin biopsies from healthy controls (HC) and patients with SSC (SSC) double stained for PEDF and $\alpha$-smooth muscle actin (SMA), or PEDF and CD31 as indicated. Arrowheads point to spindle-shaped PEDF and SMApositive cells within the dermis. For a larger field of stained skin section, see online supplementary figure 1A, B. Scale bars, $100 \mu \mathrm{m}$. (B) Dot blots show counts of PEDF-positive fibroblasts in HC and SSc samples; bars represent mean values \pm SEM ( $n=25$ microscopic fields per biopsy from five different biopsies). (C) Dot blots show blood vessel counts in HC and SSC samples; bars represent average values \pm SEM ( $n=25$ microscopic fields per biopsy from five different biopsies). Data on additional patient samples are shown in online supplementary table 1. (D) Histogram shows PEDF mRNA levels by RT-PCR in isolated HC fibroblasts (HC-FBs) and SSc fibroblasts (SSC-FBs); bars represent mean values \pm SEM ( $n=9$ samples from each $\mathrm{HC}$ and $\mathrm{SSC}$ ). Where indicated, cells were treated with $10 \mathrm{ng} / \mathrm{mL}$ TGF- $\beta$ for 24 hours; HEM, human epithelial melanocytes positive control. ${ }^{*} \mathrm{P}<0.05$, ${ }^{*} \mathrm{P}<0.01$ by unpaired t-test. (E) Western blot shows PEDF in supernatants (SUP) collected from HC-FBs and SSc-FBs cultured in the presence or absence of TGF- $\beta$ (10 ng/mL for 48 hours).

\section{RESULTS}

\section{PEDF expression is increased in SSc patient biopsies} compared with healthy donors

In healthy skin, PEDF is expressed mainly in the germinal layer of the epidermis (figure 1A). In contrast, in early diffuse SSc, we observed a strong staining for PEDF within the lower reticular dermis, both in fibroblasts and around blood vessels (figure 1A). Double IHC studies revealed that strong positivity of PEDF correlated with strong positivity of $\alpha$-SMA (figure $1 \mathrm{~A}$ and online supplementary figure $1 \mathrm{~A}, \mathrm{~B})$, indicating increased number of 
A

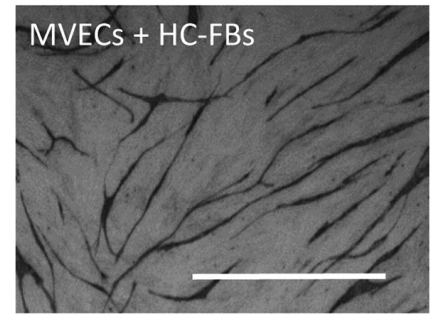

C

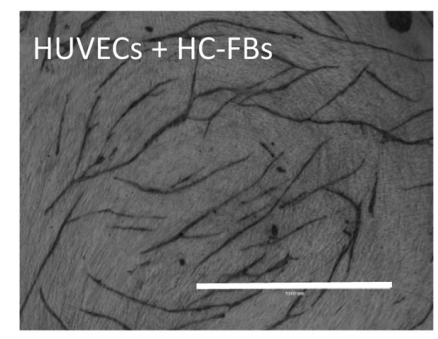

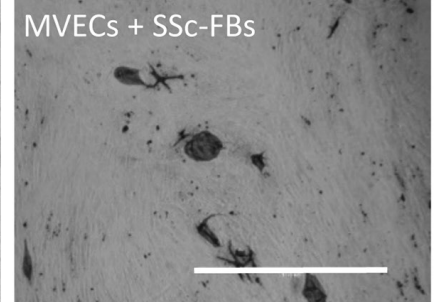

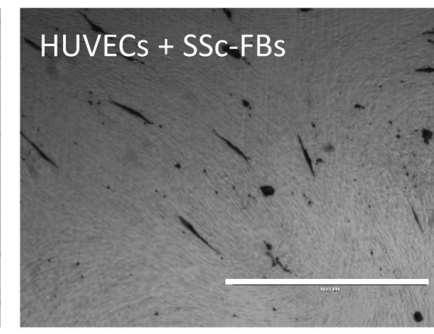

B
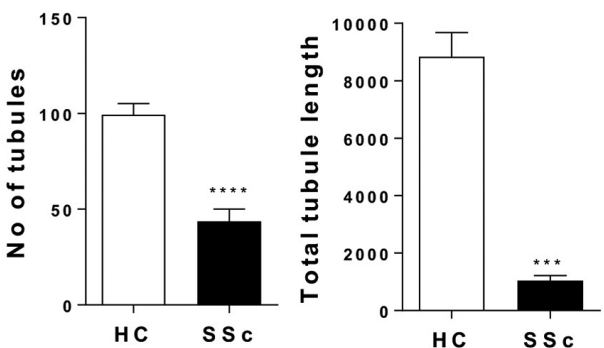

D
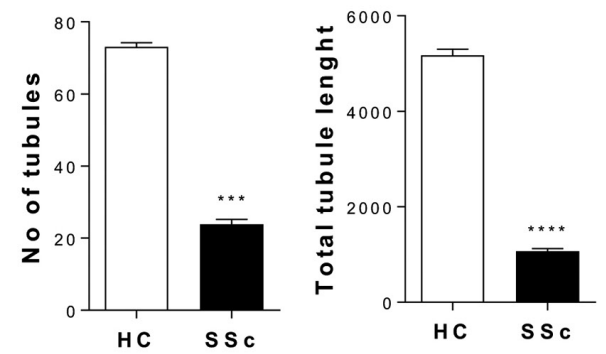

E

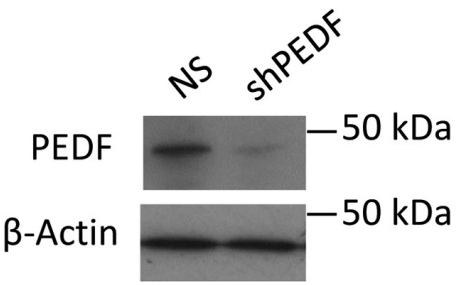

$\mathrm{F}$
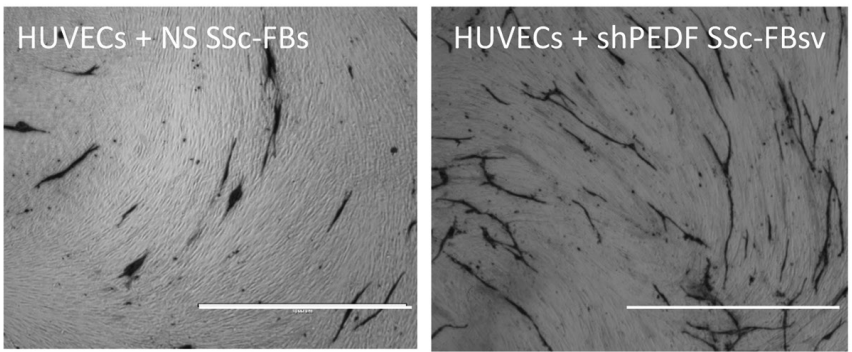

G
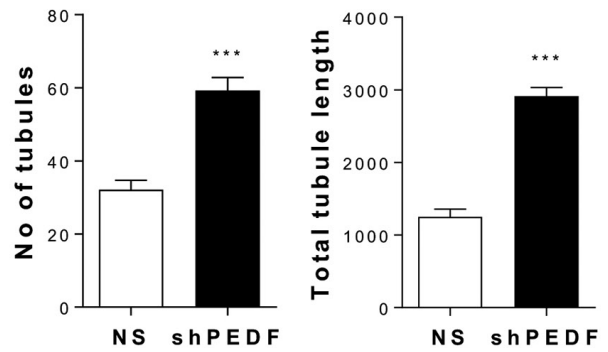

Figure 2 Suppression of angiogenesis in an organotypic co-culture assay by systemic sclerosis (SSc) fibroblasts is reversed by pigment epitheliumderived factor (PEDF) knockdown. (A, C) Images show representative microscopic fields from co-culture assays of human dermal microvascular endothelial cells (MVECs) (A) or human umbilical vein endothelial cells (HUVECs) (C) seeded onto confluent fibroblasts (FBs), healthy control (HC$\mathrm{FBs}$ ) or SSC (SSc-FBs), stained for the endothelial marker CD31 (fibroblasts are seen unstained in the background). Note that HUVECs reproduce the behaviour of MVECs in the organotypic assays. $(B, D)$ Histograms show the number of tubules and total tubule length quantified using Angiosys software, represented as mean \pm SEM ( $n=12$ microscopic fields at $\times 4$ magnification from triplicate wells). (E) Representative western blot showing intracellular PEDF levels in SSc fibroblasts treated with GolgiPlug, non-silencing control (NS) or with PEDF depletion (shPEDF) by means of lentiviral short-hairpin RNA (sh). (F) Images show representative microscopic fields from co-culture assays of HUVECs seeded onto confluent SSc fibroblasts (SSc-FBs), non-silencing control (NS) or with PEDF depletion (ShPEDF). (G) Quantification of the number of tubules and total tubule length represented as mean \pm SEM ( $n=12$ microscopic fields at $\times 4$ magnification from triplicate wells). ${ }^{*} \mathrm{P}<0.01,{ }^{* *} \mathrm{P}<0.001$ by unpaired $\mathrm{t}$-test. Scale bars, $100 \mu \mathrm{m}$.

myofibroblasts positive for PEDF in SSc biopsies. Interestingly, we also observed positivity for PEDF in vascular endothelial cells identified by CD31 staining as well as in perivascular cells (figure 1A). Quantification of PEDF-positive dermal fibroblasts within the reticular connective tissue showed significantly higher PEDF positivity in SSc fibroblasts compared with healthy control fibroblasts (figure 1B). This was accompanied by the already described decreased abundance of blood vessels in the SSc biopsies compared with healthy controls (figure 1C). Consistent with these findings, SSc skin biopsies showed 5.5 -fold increase in PEDF mRNA expression as assessed by qRT-PCR $(\mathrm{P}<0.01$, $n=5$, online supplementary figure $1 D$ ). Following this observation, we have measured PEDF concentration in 38 patients with $\mathrm{dcSSc}$ and 34 healthy controls from our observational cohort but found no statistically significant difference in serum levels of PEDF ( $\mathrm{P}=0.87)$. 
A

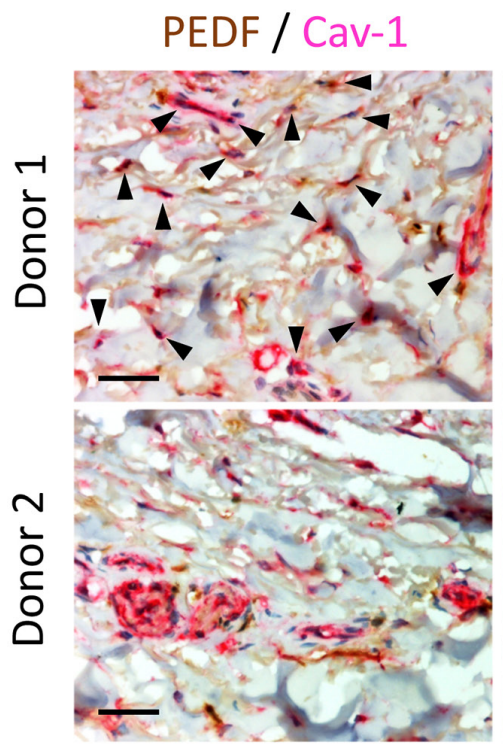

$\mathrm{HC}$

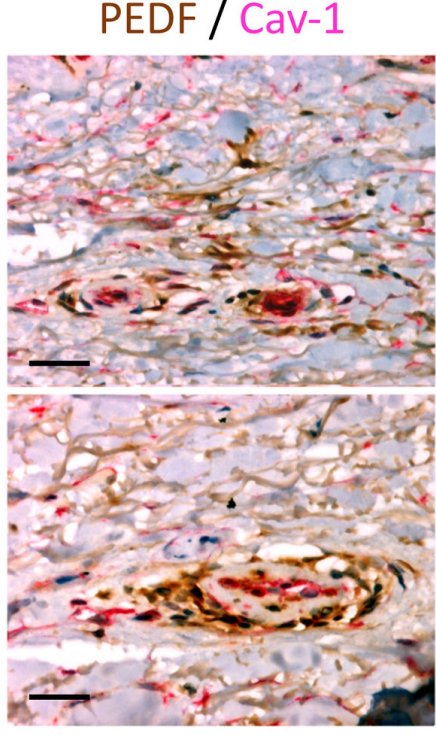

B

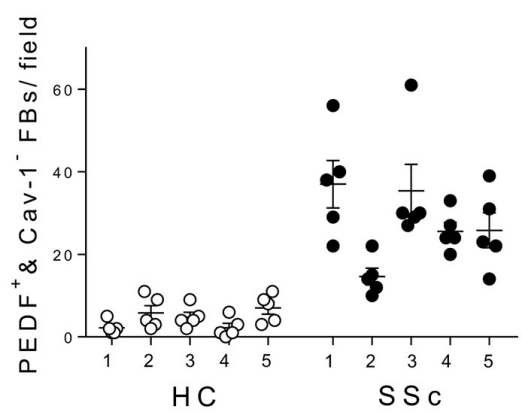

C

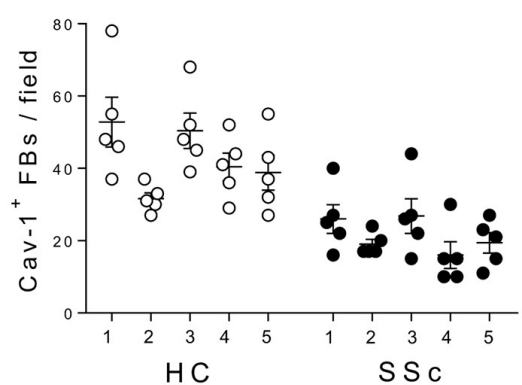

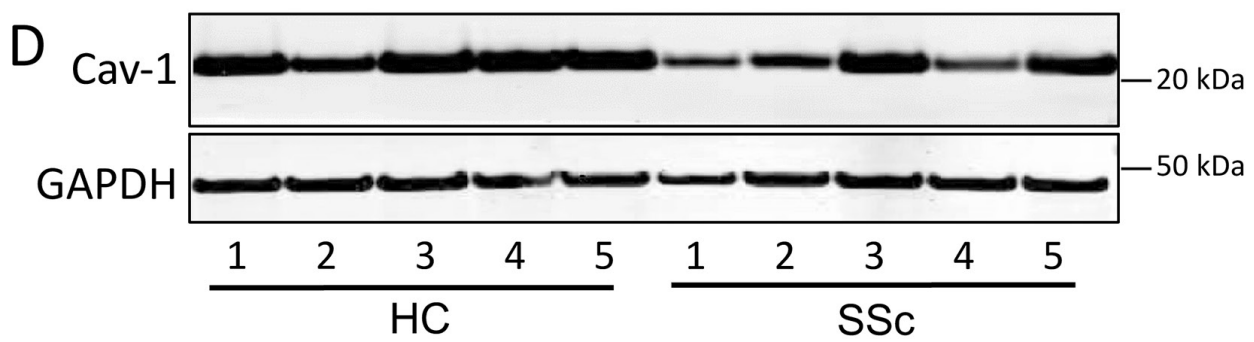

Figure 3 Decreased caveolin-1 tissue expression in systemic sclerosis (SSC) is conserved in vitro and correlates with high pigment epitheliumderived factor (PEDF) expression. (A) Images show representative forearm skin biopsies from healthy controls (HC) and patients with SSC double stained for PEDF and caveolin-1 (Cav-1). Arrowheads point to Cav-1-positive cells; note that Cav-1-positive cells show no PEDF positivity. Scale bars, $50 \mu \mathrm{m}$. (B) Dot plots show quantification of PEDF-positive and Cav-1-negative fibroblasts (FBs) from HC and patients with SSc. (C) Dot plots show quantification of Cav-1-positive FBs in HC and patients with SSc. Data on additional patient samples are shown in online supplementary table 1. (D) Western blots of five HC and SSC FB cultures for Cav-1 and glyceraldehyde 3-phosphate dehydrogenase (GAPDH). Dermal fibroblasts were subcultured from the same biopsies analysed by immunohistochemistry and loaded in the same order as shown in panels (B) and (C) and summarised in online supplementary table 1.

\section{PEDF expression in SSc and healthy fibroblasts is induced by TGF- $\beta$}

To determine whether the increase in PEDF expression resulted from upregulation in gene expression, we quantified PEDF mRNA levels by RT-PCR analysis in primary dermal FBs. FBs from patients with SSc expressed approximately 5-fold higher PEDF mRNA levels compared with HC-FBs (figure 1D). Concordantly, there was over 2-fold higher levels of intracellular PEDF protein in SSc-FBs compared with HC-FBs, as assessed by FACS mean fluorescence intensity for PEDF (online supplementary figure 1C). In order to establish whether the upregulation of PEDF expression in SSc-FBs resulted from increased TGF- $\beta$ signalling, we performed RT-PCR on healthy control and patient isolated fibroblasts following 48 hours of stimulation with hrTGF- $\beta$. hrTGF- $\beta$ induced PEDF expression in HC-FBs by 9 -fold compared with 1.6-fold increase in SSc fibroblasts (figure 1D). The levels of PEDF mRNA expression following TGF- $\beta$ stimulation were comparable in $\mathrm{HC}$ and SSc fibroblasts. Concordantly, mean fluorescent intensity by FACS for intracellular PEDF was also increased by 2.4 -fold following TGF- $\beta$ stimulation (online supplementary figure $1 \mathrm{C}$ ). Western blot analysis of supernatants from fibroblast cultures confirmed that rhTGF- $\beta$ treatment increased the levels of secreted PEDF, and this was associated with an increased secretion of collagen 1 (figure 1E) as shown previously. ${ }^{24}$

\section{SSc fibroblasts suppress angiogenesis in a PEDF-dependent manner}

To determine whether the observed increased expression of PEDF by dermal fibroblasts in SSc skin biopsies could contribute to defective angiogenesis, we performed endothelial-fibroblast organotypic angiogenesis in vitro assays in which endothelial cells form tubules highly reminiscent of capillaries formed during angiogenesis in vivo, embedded in natural matrix produced by the fibroblasts. ${ }^{21-23}$ Use of rhPEDF in this assay confirmed in vitro the known antiangiogenic effect of PEDF (online supplementary figure 2). In the same model, co-culture of MVECs with SSc-FBS showed a $60 \%$ decrease in the number of tubules and $90 \%$ decrease in total tubule length compared with HC-FBs (figure 2A, B). Similar results were obtained when HUVECs were co-cultured with HC-FBs or SSc-FBs (figure 2C, D), hence 
A

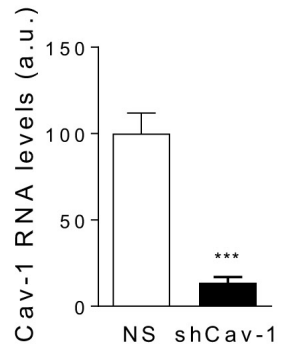

B

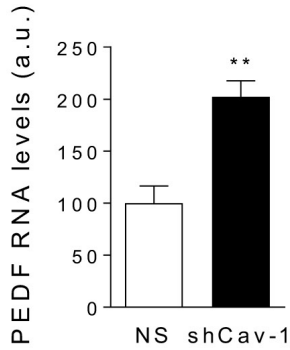

C

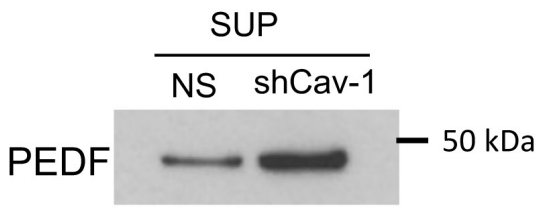

D

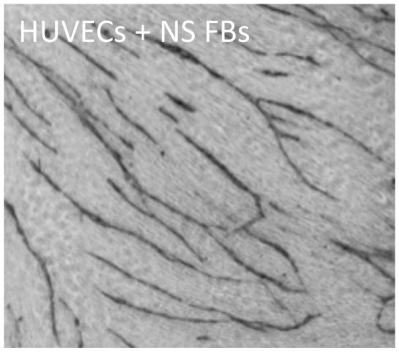

$\mathrm{F}$

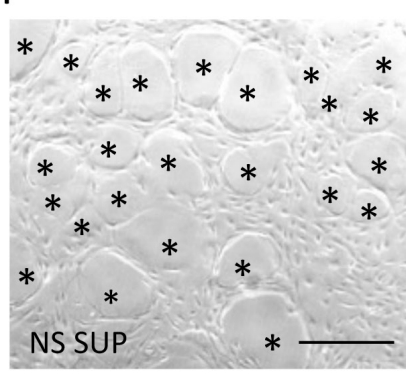

$\mathrm{H}$

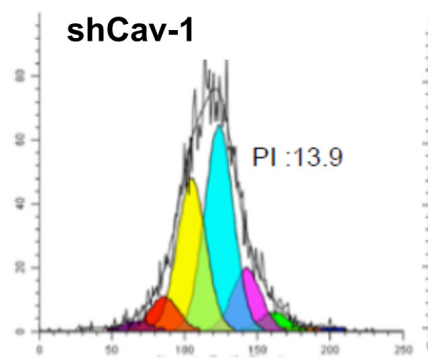

NS

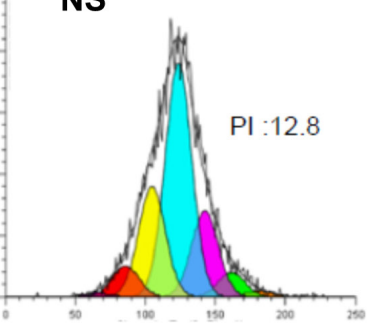

E
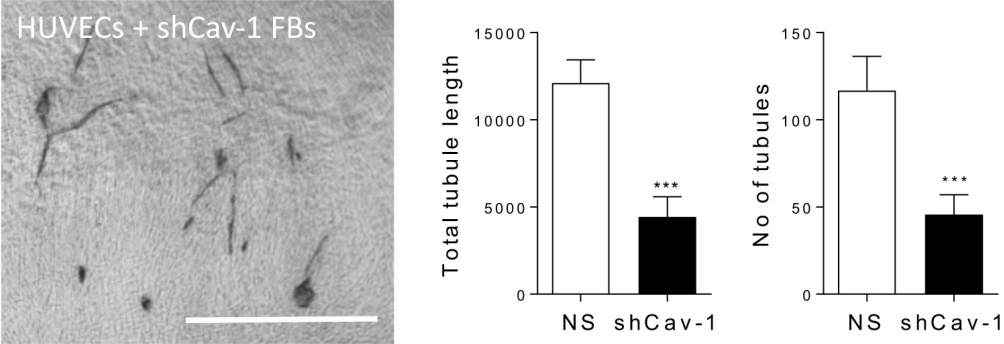

G

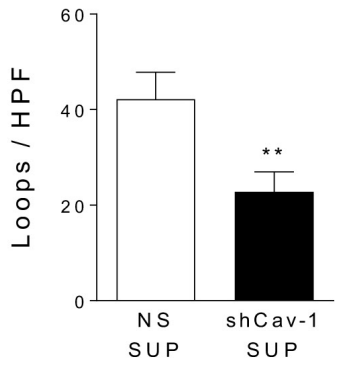

I

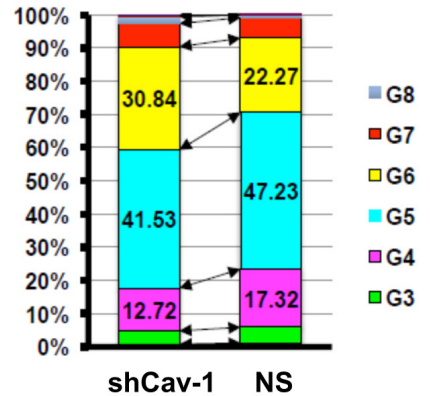

Figure 4 Caveolin-1 (Cav-1) knockdown stimulates pigment epithelium-derived factor (PEDF) expression in dermal fibroblasts and suppresses tubulogenesis without affecting endothelial cell proliferation. (A-C) Cav-1 knockdown stimulates PEDF expression. (A) Histogram depicts caveolin-1 knockdown (shCav-1) in dermal fibroblasts by means of lentiviral short-hairpin RNA (sh), as percentage non-silencing control (NS) by RT-PCR. Bars represent mean values $\pm S D$ ( $n=3$ independent experiments carried out in triplicates). ${ }^{* * *} P<0.001$ by unpaired $t$-test. (B) Histogram depicts PEDF mRNA by RT-PCR with caveolin-1 knockdown (shCav-1) compared with non-silencing control (NS). Bars represent mean values $\pm S D$ ( $n=3$ independent experiments carried out in triplicates). ${ }^{*} \mathrm{P}<0.01$ by unpaired t-test. (C) Representative western blot shows levels of PEDF in supernatants (SUP) from fibroblasts with shCav-1 compared with NS control. (D) Images show representative microscopic fields from co-culture assays of human umbilical vein endothelial cells (HUVECs) seeded onto confluent systemic sclerosis fibroblasts (SSc-FBs), non-silencing control (NS) or with PEDF depletion (shPEDF) stained by CD31. Scale bars, $100 \mu \mathrm{m}$. Note the decreased tubule formation with caveolin-1 knockdown in fibroblasts. (E) Histograms show number of tubules and total tubule length in (D) quantified using Angiosys software, represented as mean $\pm S E M$ ( $n=12$ microscopic fields from three different experiments). (F) Representative images from HUVEC matrigel assays with cultures treated with supernatants from dermal fibroblasts, non-silencing control (NS) or with caveolin-1 knockdown (shCav-1). Note the HUVEC monolayer organisation and reduced number of loops in shCav-1 supernatanttreated cultures compared with control. (G) Quantification of number of loops in (F) represented as mean $\pm S D$ ( $n=12 \mathrm{HPF}$ from three different experiments). (H) Histograms show cell generation of HUVEC preloaded with CellTrace Violet co-cultured with caveolin-1 knockdown (shCav-1) fibroblasts or non-silencing control fibroblasts (NS); Pl, relative proliferation indexes. Dye dilution analysis performed using Modfit proliferation algorithm. (I) Column chart showing quantification of HUVEC generations while in co-culture with caveolin-1 knockdown (shCav-1) fibroblasts, or non-silencing control fibroblasts (NS); chart indicates some increase in proliferation at G5/G6 in caveolin-1 knockdown fibroblast co-cultures (shCav-1) compared with non-silencing control fibroblasts (NS). ${ }^{* *} \mathrm{P}<0.01 ;{ }^{* *} \mathrm{P}<0.001$ by unpaired t-test. HPF, high power fields. 

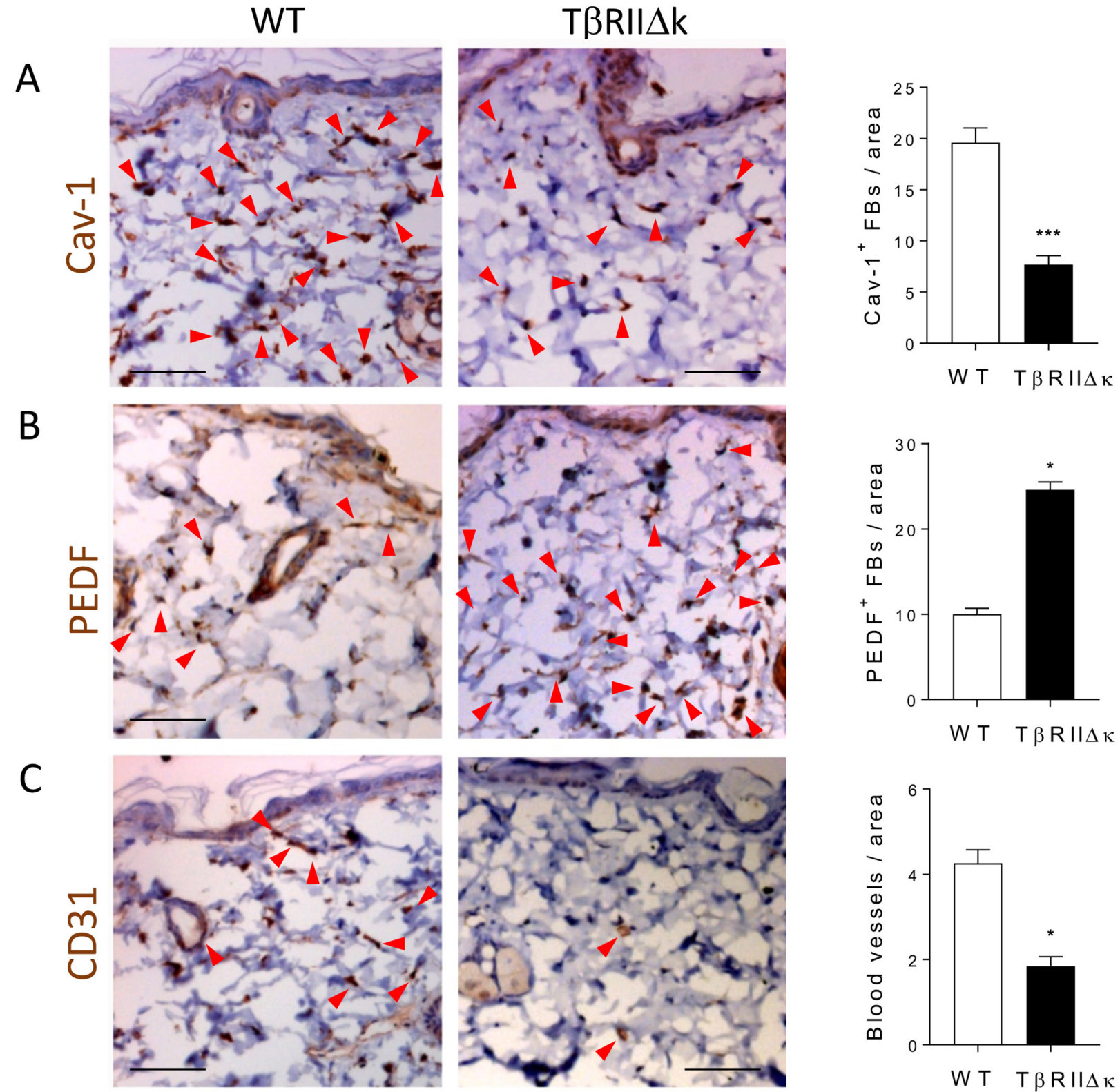

Figure 5 Skin immunohistochemistry of transgenic mice (T $\beta R I I \Delta k$-fib) and wild-type (WT) mice. Images show representative mouse skin biopsies stained for caveolin-1 (Cav-1) (A), pigment epithelium-derived factor (PEDF) (B) or CD31 (C) from transgenic T $\beta R$ II $\triangle \mathrm{k}$-fib mice and wild-type littermate controls. Note the decreased expression of caveolin-1 and increased expression of PEDF in T $\beta R I I \Delta k$-fib biopsies. Scale bar, $50 \mu m$, original magnification $\times 20$. Plots show quantification of caveolin-1-positive (A) and PEDF-positive (B) fibroblasts (FBs), and blood vessel counts (C). Bars represent average values $\pm S D$ ( $n=9$ biopsies from three mice per genotype). ${ }^{*} \mathrm{P}<0.05 ;{ }^{*}{ }^{*} \mathrm{P}<0.01 ;{ }^{* *}{ }^{*} \mathrm{P}<0.001$ by unpaired t-test.

HUVECs were employed in subsequent assays. Knockdown of PEDF in SSc-FBs by means of lentiviral shRNA (figure 2E), followed by co-culture with ECs, rescued the number of tubules and total tubule length by 1.9 -fold and 2.5 -fold, respectively, compared with SScFBs infected with control lentivirus harbouring non-silencing shRNA (figure $2 \mathrm{~F}, \mathrm{G}$ ).

\section{Downregulation of caveolin-1 induces PEDF expression in dermal fibroblasts}

TGF- $\beta$ downregulates caveolin-1 expression in vitro, both at the RNA and protein level, ${ }^{25}{ }^{26}$ and this has been associated with myofibroblasts' profibrotic activation. ${ }^{27} 28$ Double IHC studies showed an abundance of caveolin-1-positive cells largely negative for PEDF in HC skin, whereas in SSc skin biopsies, the majority of fibroblasts were PEDF positive and caveolin-1 negative (figure 3A, B). Moreover, there was an overall reduction of caveolin-1 positivity in SSc skin biopsies (figure $3 \mathrm{~A}-\mathrm{C}$ ) as previously shown. ${ }^{18}$ These results suggested an inverse correlation between PEDF and caveolin-1 expression. The increased secretion of PEDF and reduced expression of caveolin-1 was conserved in vitro. SSc fibroblasts showed increased PEDF protein levels (online supplementary figure 1) and decreased expression of caveolin-1 (figure 3D). Interestingly, the relative expression of caveolin-1 in subcultured fibroblasts (SSc 1-5) followed the same trend observed by IHC in patients 1-5 (online supplementary table 1 ). Stable silencing of caveolin-1 by lentiviral delivery of caveolin-1 shRNA showed $85 \%$ caveolin-1 knockdown compared with control (figure 4A). Fibroblasts with caveolin-1 knockdown displayed over 2-fold upregulation of PEDF expression compared with controls (figure 4B). 


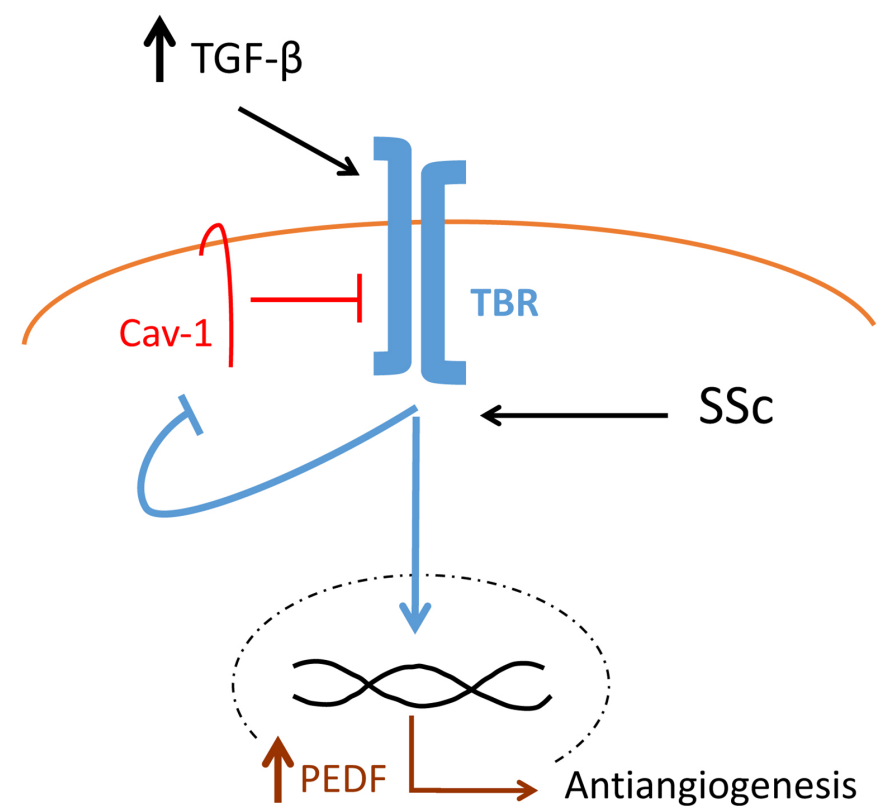

Figure 6 Mechanistic model depicting the relationship between caveolin-1 (Cav-1) and pigment epithelium-derived factor (PEDF). Transforming growth factor beta (TGF- $\beta$ ) signalling strength is autoregulated by Cav-1-dependent TGF- $\beta$ receptor internalisation. ${ }^{40}$ Caveolin-1 downregulation and potentiation of TGF- $\beta$ signalling promotes PEDF transcription, expression and secretion by dermal fibroblasts, suppressing angiogenesis in systemic sclerosis (SSc). Exposure to high levels of TGF- $\beta$ and receptor overactivation in SSC sustains caveolin-1 downregulation at the transcriptional level, ${ }_{1}^{27}$ thus promoting further PEDF expression and impairment of angiogenesis.

Accordingly, we observed an increased secretion of PEDF in the supernatants harvested from fibroblasts with caveolin-1 knockdown compared with control (figure 4C). These results show that decreased caveolin-1 expression in dermal fibroblasts induces the expression and secretion of PEDF in vitro and that the inverse correlation of expression is observable in vivo.

\section{Caveolin-1 downregulation in fibroblasts inhibits angiogenesis without affecting endothelial viability and proliferation}

Fibroblasts with lentiviral shRNA driven caveolin-1 knockdown suppressed tubule formation in the organotypic angiogenesis assay, with a $62.5 \%$ decrease in tubule length and $58.2 \%$ decrease in total number of tubules $(\mathrm{P}<0.001$ for both) (figure 4D) compared with controls. Accordingly, matrigel assays using supernatants harvested from the same cells showed nearly $50 \%$ reduction in tube formation $(\mathrm{P}<0.01)$ (figure $4 \mathrm{~F}, \mathrm{G})$. Therefore, the inhibitory effect on tubulogenesis of caveolin-1 knockdown in fibroblasts is transferable by tissue culture supernatants, supporting the notion that it is mediated via secreted factors such as PEDF. Consistently, immunodepletion of PEDF from fibroblast supernatants, through treatment with a blocking antibody (online supplementary figure 3A), rescued tubule morphogenesis significantly in the matrigel assay (online supplementary figure $3 \mathrm{~B}, \mathrm{C}$ ).

Viability of endothelial cells (CD31 $1^{\text {pos }} / \mathrm{CD} 90^{\text {neg }}$ ) in the organotypic angiogenesis assay was comparable between the two experimental conditions (online supplementary figure 3D). Similarly, the proliferation index by the dye dilution method of endothelial cells was comparable in the two conditions (figure $4 \mathrm{H}$, I). These data indicate that the impairment in angiogenesis, mediated by fibroblasts with caveolin-1 knockdown, does not result from decreased endothelial cell viability or proliferation.

\section{TGF- $\beta$ signalling suppresses caveolin-1 expression and stimulates PEDF expression in vivo}

To investigate whether the findings from the patient sample analysis and culture systems hold in an in vivo model of overactivation of TGF- $\beta$ signalling, we employed mice with controlled overexpression of TGF- $\beta$ receptors in fibroblasts driven by the col1a1 promoter (T $\beta$ RII $\Delta \mathrm{k}$-fib). Analysis of sections from mouse skin biopsies showed reduced caveolin-1 expression in T $\beta R I I \Delta k$-fib transgenic mice compared with wild-type littermate controls (figure 5A). Conversely, expression of PEDF was increased in the skin of the transgenic mice (figure 5B). Importantly, increased PEDF and reduced caveolin-1 expression in T $\beta$ RII $\Delta \mathrm{k}$-fib transgenic mice was associated with a reduction in the number of capillaries as assessed by IHC for CD31 (figure 5C).

\section{DISCUSSION}

Here, we demonstrate for the first time that dermal fibroblasts in SSc play a direct role in the impairment of angiogenesis via secretion of PEDF. The increased PEDF expression in dcSSc skin biopsies validates the proteomics data of dcSSc fibroblast secretome, ${ }^{8}$ and it is consistent with the increased PEDF expression observed in IPF by Cosgrove et al. ${ }^{17}$ The PEDF-positive cells were both tissue fibroblasts (for their classic spindle-shape morphology) and myofibroblasts (SMA positive). In addition, we observed high PEDF expression in cells with perivascular localisation, suggesting that in vivo, multiple cell types may contribute to aberrant secretion of PEDF in SSc. Nevertheless, we did not find an increased PEDF serum concentration in patients with dcSSc versus healthy controls, consistent with the known paracrine mode of action of PEDF.

Interestingly, PEDF was found significantly more abundant in the lower dermis of patients with dcSSc, suggesting that reticular fibroblasts may play an important role in the initiation and progression of impaired angiogenesis.

Here, we show for the first time that TGF- $\beta$ signalling in fibroblasts suppresses angiogenesis through secretion of PEDF, and that this pathway remains active in fibroblasts explanted from SSc skin. This is consistent with a wealth of data in the literature indicating that SSc fibroblasts maintain in vitro hallmarks of TGF- $\beta$ signalling activation with passaging, including increased collagen production, phosphorylation of SMAD, Jnk and ERK as well as increased expression of $\alpha$-SMA.

Another big set of evidence indicates that PEDF mediates its antiangiogenic effects through multiple mechanisms including suppression of migration via p38 signalling, induction of apoptosis through MEK5/Erk5 signalling to peroxisome proliferator-activated receptor gamma and NF- $\mathrm{BB},{ }^{9} 2930$ and antagonism of vascular endothelial growth factor (VEGF) signalling via $\gamma$-secretase cleavage of VEGF receptors. ${ }^{13} 31$

Therefore, our data link two widely demonstrated molecular pathways and suggest an explanation of TGF- $\beta$-induced vasculopathy in SSc.

Intriguingly, PEDF has been found to have an antifibrotic effect in a chemically induced model of liver fibrosis. ${ }^{32} 33$ Although we cannot exclude that the TGF- $\beta$-induced expression of PEDF observed both in $\mathrm{SSc}^{834}$ and idiopathic pulmonary fibrosis ${ }^{17}$ could represent an attempt to negatively feedback the fibrotic process, here we show that PEDF expression can ultimately contribute to the defective angiogenesis in a paracrine manner during SSc. 
Previously, we and others reported that SSc skin and lung biopsies show a decreased expression of caveolin-1 when compared with healthy controls, which is associated with tissue fibrosis. ${ }^{1825-28}$

Caveolin-1 plays a bidirectional role in endothelial cells during angiogenesis (reviewed in Sowa ${ }^{35}$ ) by promoting ${ }^{36}{ }^{37}$ or inhibiting ${ }^{38}$ blood vessel formation; however, the indirect effects on angiogenesis of low caveolin-1 levels in tissue fibroblasts were never studied before. The current study shows that similar to previous data on $\alpha$-SMA and collagen, also PEDF increased expression in vivo is conserved in subcultured dermal fibroblasts consistent with the known sustained TGF- $\beta$ signalling of SSc fibroblasts in vitro. Most importantly, we show that the inverse correlation in the expression of caveolin-1 and PEDF is associated with the decreased number of capillaries observed in SSc. Further, we show a causative link between caveolin-1 decreased expression and PEDF secretion, which in turn suppresses angiogenesis in vitro, without affecting cell viability or proliferation. Altogether, the rescue experiments we have performed using PEDF knockdown in SSc fibroblasts and PEDF blocking antibody in SSc supernatants (figure 2F, G and online supplementary figure 3 ) clearly show the importance of PEDF in the antiangiogenic phenotype of these cells.

Further, here we show that the inverse relationship between caveolin-1 and PEDF expression observed in the patient samples is recapitulated in the T $\beta R I I \Delta \mathrm{k}$-fib transgenic mice with overactivation of TGF- $\beta$ signalling in tissue fibroblasts. ${ }^{7}$ Consistent with this observation and the antiangiogenic function of PEDF, we also noted a reduced capillary density in the TRRII $\Delta \mathrm{k}$-fib mouse skin biopsies. Functional experiments will be necessary to determine whether this transgenic line can be used as preclinical model of TGF- $\beta$-induced vasculopathy and targeting of this signalling axis in SSc.

Overall, the findings in this study strongly support the notion that TGF- $\beta$ is involved in the pathogenesis of vasculopathy in SSc, and establish a causative link between caveolin-1 downregulation in tissue fibroblasts, PEDF expression and defective angiogenesis (figure 6). While this study focuses on dcSSc, in follow-up studies it would be interesting to investigate the relationship between PEDF, caveolin-1 and angiogenesis in limited cutaneous SSc (lcSSc). It is worth noting that lcSSc skin biopsies do not show the hallmarks of TGF- $\beta$ activation seen in dcSSc, ${ }^{39}$ and therefore it would be very interesting to investigate whether the vasculopathy observed in lcSSc is driven by a non-TGF- $\beta$-related pathway.

\footnotetext{
Author affiliations

'Leeds Institute of Rheumatic and Musculoskeletal Medicine, University of Leeds, Leeds, UK

${ }^{2}$ Department of Biotechnological and Applied Clinical Science, Rheumatology Unit, School of Medicine, University of L'Aquila, L'Aquila, Italy

${ }^{3}$ School of Pharmacy and Medical Sciences, University of Bradford, Bradford, UK

${ }^{4}$ Clinical Pathology Department, Faculty of Medicine, Mansoura University, Mansoura, Egypt

${ }^{5}$ NIHR Leeds Musculoskeletal Biomedical Research Centre, Leeds Teaching Hospital NHS Trust, Leeds, UK

${ }^{6}$ Signal Transduction and Tumour Microenvironment Group, Leeds Institute of Cancer and Pathology, University of Leeds, Leeds, UK

${ }^{7}$ Rheumatology Department of Lucania, Rheumatology Institute of Lucania (IReL), San Carlo Hospital of Potenza and Madonna delle Grazie Hospital of Matera, Potenza, Italy

${ }^{8}$ Rheumatology Institute of Lucania (IReL), San Carlo Hospital of Potenza, Potenza, Italy

${ }^{9}$ Centre for Rheumatology and Connective Tissue, UCL Medical School Royal Free Campus, London, UK

${ }^{10}$ Leeds Institute of Cancer and Pathology, University of Leeds, Leeds, UK
}

Contributors VL, JEC, YME-S, MS, GG, ECD-S and FE contributed to the experimental data collection and analysis. GA and PC contributed to the identification and collection of patient clinical data and biosamples. PE, CPD, RG and GM contributed to data revision, manuscript revision and discussion. VL and JEC drafted the manuscript. FDG ideated the experimental plan and supervised all aspects of research. FDG and GM critically reviewed data and their analysis.

Funding This study was partially funded by NIHR CDF to FDG and EULAR ODP grant to FDG.

\section{Competing interests None declared.}

Ethics approval The study was approved by the NHS REC 10/H1306/88 and Institutional Review Board of University of L'Aquila.

Provenance and peer review Not commissioned; externally peer reviewed.

Data sharing statement There are no additional or unpublished data that need to be shared as part of this study.

Open Access This is an Open Access article distributed in accordance with the Creative Commons Attribution Non Commercial (CC BY-NC 4.0) license, which permits others to distribute, remix, adapt, build upon this work non-commercially, and license their derivative works on different terms, provided the original work is properly cited and the use is non-commercial. See: http://creativecommons.org/ licenses/by-nc/4.0/

(c) Article author(s) (or their employer(s) unless otherwise stated in the text of the article) 2018. All rights reserved. No commercial use is permitted unless otherwise expressly granted.

\section{REFERENCES}

1 LeRoy EC. Systemic sclerosis. A vascular perspective. Rheum Dis Clin North Am 1996;22:675-94.

2 Denton CP, Khanna D. Systemic sclerosis. Lancet 2017;390:1685-99.

3 Liakouli V, Cipriani P, Marrelli A, et al. Angiogenic cytokines and growth factors in systemic sclerosis. Autoimmun Rev 2011;10:590-4.

4 Cabral-Marques O, Riemekasten G. Vascular hypothesis revisited: role of stimulating antibodies against angiotensin and endothelin receptors in the pathogenesis of systemic sclerosis. Autoimmun Rev 2016;15:690-4.

5 Del Galdo F, Lisanti MP, Jimenez SA. Caveolin-1, transforming growth factor- $\beta$ receptor internalization, and the pathogenesis of systemic sclerosis. Curr Opin Rheumatol 2008;20:713-9.

6 Lafyatis R. Transforming growth factor $\beta$-at the centre of systemic sclerosis. Nat Rev Rheumatol 2014;10:706-19.

7 Derrett-Smith EC, Dooley A, Gilbane AJ, et al. Endothelial injury in a transforming growth factor $\beta$-dependent mouse model of scleroderma induces pulmonary arterial hypertension. Arthritis Rheum 2013;65:2928-39.

8 Del Galdo F, Shaw MA, Jimenez SA. Proteomic analysis identification of a pattern of shared alterations in the secretome of dermal fibroblasts from systemic sclerosis and nephrogenic systemic fibrosis. Am J Pathol 2010;177:1638-46.

9 Dawson DW, Volpert OV, Gillis P, et al. Pigment epithelium-derived factor: a potent inhibitor of angiogenesis. Science 1999;285:245-8.

10 Liu H, Ren JG, Cooper WL, et al. Identification of the antivasopermeability effect of pigment epithelium-derived factor and its active site. Proc Natl Acad Sci U S A 2004;101:6605-10.

11 Tombran-Tink J, Johnson LV. Neuronal differentiation of retinoblastoma cells induced by medium conditioned by human RPE cells. Invest Ophthalmol Vis Sci 1989:30:1700-7

12 Polato F, Becerra SP. Pigment epithelium-derived factor, a protective factor for photoreceptors in vivo. Adv Exp Med Biol 2016;854:699-706.

13 Matsui T, Nishino Y, Maeda S, et al. PEDF-derived peptide inhibits corneal angiogenesis by suppressing VEGF expression. Microvasc Res 2012;84:105-8.

14 Cai J, Jiang WG, Grant MB, et al. Pigment epithelium-derived factor inhibits angiogenesis via regulated intracellular proteolysis of vascular endothelial growth factor receptor 1. J Biol Chem 2006;281:3604-13.

15 Mori K, Duh E, Gehlbach P, et al. Pigment epithelium-derived factor inhibits retinal and choroidal neovascularization. J Cell Physiol 2001;188:253-63.

16 Park K, Jin J, Hu Y, et al. Overexpression of pigment epithelium-derived factor inhibits retinal inflammation and neovascularization. Am J Pathol 2011;178:688-98

17 Cosgrove GP, Brown KK, Schiemann WP, et al. Pigment epithelium-derived factor in idiopathic pulmonary fibrosis: a role in aberrant angiogenesis. Am J Respir Crit Care Med 2004; 170:242-51.

18 van den Hoogen F, Khanna D, Fransen J, et al. 2013 classification criteria for systemic sclerosis: an American College of Rheumatology/European League Against Rheumatism collaborative initiative. Ann Rheum Dis 2013;72:1747-55.

19 Del Galdo F, Sotgia F, de Almeida CJ, et al. Decreased expression of caveolin 1 in patients with systemic sclerosis: crucial role in the pathogenesis of tissue fibrosis. Arthritis Rheum 2008;58:2854-65. 
20 Piera-Velazquez S, Louneva N, Fertala J, et al. Persistent activation of dermal fibroblasts from patients with gadolinium-associated nephrogenic systemic fibrosis. Ann Rheum Dis 2010;69:2017-23.

21 Bishop ET, Bell GT, Bloor S, et al. An in vitro model of angiogenesis: basic features. Angiogenesis 1999;3:335-44.

22 Hetheridge C, Mavria G, Mellor H. Uses of the in vitro endothelial-fibroblast organotypic co-culture assay in angiogenesis research. Biochem Soc Trans 2011;39:1597-600.

23 Mavria G, Vercoulen Y, Yeo M, et al. ERK-MAPK signaling opposes Rho-kinase to promote endothelial cell survival and sprouting during angiogenesis. Cancer Cell 2006;9:33-44.

24 Alcantara MB, Nemazannikova N, Elahy M, et al. Pigment epithelium-derived factor upregulates collagen I and downregulates matrix metalloproteinase 2 in osteosarcoma cells, and colocalises to collagen I and heat shock protein 47 in fetal and adult bone. J Pharm Pharmacol 2014;66:1586-92.

25 Tourkina E, Bonner M, Oates J, et al. Altered monocyte and fibrocyte phenotype and function in scleroderma interstitial lung disease: reversal by caveolin-1 scaffolding domain peptide. Fibrogenesis Tissue Repair 2011;4:15.

26 Tourkina E, Richard M, Oates J, et al. Caveolin-1 regulates leucocyte behaviour in fibrotic lung disease. Ann Rheum Dis 2010;69:1220-6.

27 Sanders YY, Cui Z, Le Saux CJ, et al. SMAD-independent down-regulation of caveolin- 1 by TGF- $\beta$ : effects on proliferation and survival of myofibroblasts. PLoS One 2015;10:e0116995.

28 Xia H, Khalil W, Kahm J, et al. Pathologic caveolin-1 regulation of PTEN in idiopathic pulmonary fibrosis. Am J Pathol 2010;176:2626-37.

29 Orgaz JL, Ladhani O, Hoek KS, et al. Loss of PEDF enables migration, invasion and metastatic spread of human melanoma. Oncogene 2009;28:4147-61.
30 Biyashev D, Veliceasa D, Kwiatek A, et al. Natural angiogenesis inhibitor signals through Erk5 activation of peroxisome proliferator-activated receptor gamma (PPARgamma). J Biol Chem 2010;285:13517-24.

31 Ablonczy Z, Prakasam A, Fant J, et al. Pigment epithelium-derived factor maintains retinal pigment epithelium function by inhibiting vascular endothelial growth factor-R2 signaling through gamma-secretase. J Biol Chem 2009;284:30177-86.

32 Tsai TH, Shih SC, Ho TC, Tc H, et al. Pigment epithelium-derived factor 34-mer peptide prevents liver fibrosis and hepatic stellate cell activation through down-regulation of the PDGF receptor. PLoS One 2014;9:e95443.

33 Ho TC, Chen SL, Shih SC, et al. Pigment epithelium-derived factor is an intrinsic antifibrosis factor targeting hepatic stellate cells. Am J Pathol 2010;177:1798-811.

34 Głodkowska-Mrówka E, Górska E, Ciurzyński M, et al. Pro- and antiangiogenic markers in patients with pulmonary complications of systemic scleroderma. Respir Physiol Neurobiol 2015;209:69-75.

35 Sowa G. Caveolae, caveolins, cavins, and endothelial cell function: new insights. Front Physiol 2012;2:120.

36 Chang SH, Feng D, Nagy JA, et al. Vascular permeability and pathological angiogenesis in caveolin-1-null mice. Am J Pathol 2009;175:1768-76.

37 Tahir SA, Yang G, Goltsov AA, et al. Tumor cell-secreted caveolin-1 has proangiogenic activities in prostate cancer. Cancer Res 2008;68:731-9.

38 Lin MI, Yu J, Murata T, et al. Caveolin-1-deficient mice have increased tumor microvascular permeability, angiogenesis, and growth. Cancer Res 2007;67:2849-56.

39 Milano A, Pendergrass SA, Sargent JL, et al. Molecular subsets in the gene expression signatures of scleroderma skin. PLoS One 2008;3:e2696.

40 Del Galdo F, Lisanti MP, Jimenez SA. Caveolin-1, transforming growth factor-beta receptor internalization, and the pathogenesis of systemic sclerosis. Curr Opin Rheumatol 2008;20:713-9. 